\title{
M. Łaziński, Wyktady o aspekcie polskiego czasownika, Wydawnictwa Uniwersytetu Warszawskiego, Warszawa 2020, pp. 315.
}

La monografia di Marek Łaziński, concepita come un corso universitario dedicato all'aspetto verbale del polacco, è un evento importante per la linguistica polacca e slava, anche perché vede la luce diversi decenni dopo gli studi di Koschmieder (Nauka o aspektach czasownika polskiego w zarysie. Próba syntezy, Wilno 1934) e di Piernikarski (Typy opozycji aspektowych czasownika polskiego na tle stowiańskim, Wrocław 1969).

L'aspetto verbale polacco viene descritto in questo lavoro con frequenti riferimenti alle altre lingue slave e ai numerosi importanti contributi teorici su questo tema prodotti a livello mondiale e in ambito slavistico dedicati principalmente al russo. Fin dall'introduzione l'A. anticipa che il funzionamento dell'aspetto è determinato da una serie di parametri, spesso in concorrenza tra loro, che vengono sviluppati nella trattazione successiva. Il volume è organizzato in sei capitoli che si concludono con la presentazione di un algoritmo degli usi delle forme aspettuali sulla base dell'interazione di diversi fattori. Il libro si chiude con un' interessante appendice divisa in due parti intitolate rispettivamente Lineamenti soggettivi della storia degli studi aspettologici e L'aspetto nei dizionari, quest'ultima dedicata alla presentazione delle forme aspettuali nei dizionari polacchi e ai suggerimenti per la loro etichettatura nei corpora.

Il cap. I in cui si tratta dell'opposizione aspettuale, delle sue marche formali, dei parametri semantici e della statistica d'uso delle varie forme, vera novità di questo lavoro, è concepito come una sorta di lezione introduttiva e potrebbe anche essere considerato come una lezione unica, una sorta di corso base. I singoli argomenti, presentati alla luce delle diverse teorie, vengono approfonditi nei capitoli successivi.

Così, il cap. II si apre con considerazioni sul rapporto tra tempo e aspetto e le sue conseguenze diversificate nell'uso aspettuale nelle lingue slave, soprattutto per quanto riguarda l'espressione del presente perfettivo. Per l'A. “il tempo grammaticale determina lo scorrere del tempo logico, mentre l'aspetto determina la maniera in cui una situazione si svolge nel tempo" (p. 54). Viene poi delineata l'origine dell'aspetto polacco e slavo nato dall'evoluzione del sistema dei tempi grammaticali e dai processi derivazionali di prefissazione e di suffissazione che modificano il significato dei verbi dando luogo ai vari modi dell'azione (Aktionsarten) passati in rassegna ed associati ai singoli prefissi. Insieme ai prefissi verbali e alla loro funzione nella formazione delle coppie aspettuali, viene esaminata anche la suffissazione e il suo ruolo nel fenomeno delle cosiddette tròjki (terzetti) aspettuali. 
Nel cap. III vengono affrontate le classi azionali (semantiche) dei verbi e viene discusso l'adattamento della nota classificazione di Vendler alle peculiarità delle lingue slave. Si passa quindi all'esame dei verbi di moto che in molte lingue formano una classe distinta mentre in quelle slave hanno anche svolto un ruolo nello sviluppo dell'aspetto. Oltre a presentare le caratteristiche relative alla (in)determinatezza del movimento, alla deissi o alla sua mancanza, l'A. prende in esame i verbi di moto alla luce della classificazione semantica in path verbs e manner verbs, verb framed e satellite framed verbs, proposta negli ultimi decenni dalla tipologia linguistica di stampo cognitivista (Talmy, Toward a Cognitive Semantics, 2000).

Il cap. IV è dedicato alla relazione tra le classi semantiche e il contesto da una parte, e la scelta delle forme perfettive od imperfettive dall'altra; l'interazione tra questi fattori dà luogo a vari tipi di coppie aspettuali. Nella ricerca dell' invariante delle due forme aspettuali l'A. assegna il valore fattivo concreto al perfettivo che identifica l'evento, mostrando anche come i significati subalterni all' invariante perfettivo distinguano il polacco dalle altre lingue slave. Rinuncia invece ad identificare l'invariante dell' imperfettivo in quanto polisemico, distinguendone i valori più specifici quali durativo, attuale permanente, iterativo, potenziale, fattivo generale, presente storico e scenico, performativo e definitorio, di cui gli ultimi due raramente sono trattati negli studi aspettologici.

Nel cap. $\mathrm{v}$ vengono esaminate le funzioni e gli usi delle forme aspettuali finite ed infinite e i dati sulle loro occorrenze nel Corpus Nazionale della Lingua Polacca. Il ricorso ai corpora costituisce una vera novità nella descrizione dell'aspetto in polacco, un vero e proprio banco di prova per le descrizioni teoriche. In questo capitolo viene anche messo in evidenza come le forme aspettuali verbali finite ed infinite (queste ultime poco descritte nei lavori sull'aspetto) siano condizionate dalla semantica delle situazioni e come assumano una funzione narrativa: il perfettivo indica i confini degli eventi, mentre l'imperfettivo gli stati o i concatenamenti degli eventi. Questa parte contiene anche considerazioni sull'aspetto e sulla modalità con qualche accenno al fenomeno della negazione. Interessanti osservazioni riguardano, inoltre, l'uso delle forme aspettuali nelle costruzioni verbali spesso neglette nell'uso ufficiale del polacco, anche se piuttosto frequenti nel registro colloquiale, come il quasi-perfetto (mam to zrobione), l'assentivo (bytam się dowiedzieć), l'aspetto di nomina actionis e la loro distribuzione. Le conclusioni riguardo all'uso delle singole forme aspettuali, basate sui dati del corpus, registrano in maniera oggettiva le conseguenti tendenze della lingua.

Il cap. vi offre un'importante illustrazione dell'utilità dell'analisi semantica dell'aspetto verbale applicata al linguaggio dei testi giuridici polacchi, che appare cruciale per una corretta interpretazione degli articoli dei Codici. Questa interessante analisi viene condotta anche a livello comparato con altre lingue slave e con il tedesco, i cui Codici, per ragioni storiche, hanno avuto un' influenza su quelli polacchi.

Nel cap. viI sono presentate le conclusioni sotto forma di un algoritmo dell'uso aspettuale in polacco, che prende in considerazione solo le forme finite dei verbi e rappresenta, per necessità, una semplificazione dell'esposizione precedente. I parametri che determinano le scelte delle forme aspettuali sono le classi azionali, l'iteratività, il registro (narrativo o discorsivo) ed infine i condizionamenti testuali, come il modo condizionale e la negazione (p. 234). La prima distinzione riguarda la categoria dell' iteratività: se l'azione del verbo è singola o non definita da questo punto di vista, la scelta aspettuale avviene secondo una serie di parametri complessi: classe azionale, registro, semantica della situazione (raggiungimento o meno del cambiamento e dello stato risultante), o ancora grammaticali (tempo, modo) e pragmatici (ad es. negazione). L'azione iterativa comprende invece da una parte le situazioni abituali e non delimitate, espresse dalle forme imperfettive, mentre dall'altra, le situazioni limitate o 'esemplificanti' che possono essere espresse da entrambi gli aspetti (par. 4.2.). 
Il libro si chiude con un'appendice sullo stato dell'arte degli studi e sulla loro storia (panorama di cui si sottolinea nel titolo della prima il taglio "soggettivo"), che normalmente, come riconosce l'A. stesso, dovrebbe aprire una monografia dedicata all'aspetto e non essere collocata alla fine. Tale scelta viene giustificata con il fatto che l'aspettologia rappresenta un campo d'indagine così variegato, con una ricchezza teorica e terminologica spesso non condivisa, da richiedere una conoscenza preliminare del fenomeno e dei diversi approcci alla descrizione, per capirne la storia (p. 237). La seconda parte dell'appendice, dedicata a come l'aspetto viene trattato nei dizionari, affronta la spinosa questione del criterio dell' individuazione della coppia aspettuale e la conseguente decisione su quale membro della coppia vada considerato come basico, come forma di citazione nella redazione sia nei dizionari monolingui, sia nei più recenti dizionari bilingui o in altri ancora. Diversi dizionari ormai hanno adottato un approccio più avanzato nella presentazione delle coppie aspettuali e nella scelta delle forme basiche, individuate in base alla classe azionale di appartenenza del verbo.

L'appendice si conclude con delle considerazioni sull'aspetto nei corpora linguistici che riconoscono correttamente i valori aspettuali dei lemmi, ma riscontrano difficoltà nei casi di omonimia (come ad es. in pochodzic ${ }^{1}$ - 'provenire' e pochodzic ${ }^{2}$ - 'camminare un po'). Inoltre, appare problematica la ricerca delle opposizioni aspettuali nei corpora, data l'assenza di mezzi per identificare i membri della coppia, ostacolata anche dall'esistenza dei perfectiva ed imperfectiva tantum. Viene quindi illustrata la soluzione adottata dal gruppo dell'Università di Varsavia (con la partecipazione dell'A.) e quello di Magonza che lavora al corpus polacco-tedesco. Nel corpus vengono inseriti i tags che si riferiscono alle coppie aspettuali, nonché l'informazione sulle caratteristiche formali prefissali o suffissali di tali coppie (p. 272). Grazie a questo sistema si possono condurre ricerche con metodi nuovi, più semplici rispetto al passato.

Nel complesso si tratta di un'opera molto ricca, difficile da presentare in maniera esauriente nello spazio limitato di una recensione. Il lavoro è arricchito da puntuali considerazioni terminologiche, molto utili, dato che la frequente variazione terminologica negli studi sull'aspetto rende più difficoltosa la fruizione delle descrizioni. Il libro è prezioso sia perché presenta da diverse angolazioni lo stato della ricerca e le sue applicazioni, sia perché apre nuove prospettive grazie all'uso statistico degli strumenti elettronici per la descrizione dell'aspetto. 